\title{
Investigation of Trap States in Mid-Wavelength Infrared Type II Superlattices Using Time-Resolved Photoluminescence
}

\author{
BLAIR C. CONNELLY, ${ }^{1,3}$ GRACE D. METCALFE, ${ }^{1}$ HONGEN SHEN, ${ }^{1}$ \\ MICHAEL WRABACK,${ }^{1}$ CHADWICK L. CANEDY, ${ }^{2}$ IGOR VURGAFTMAN, ${ }^{2}$ \\ JOSEPH S. MELINGER, ${ }^{2}$ CHAFFRA A. AFFOUDA, ${ }^{2}$ ERIC M. JACKSON, ${ }^{2}$ \\ JILL A. NOLDE, ${ }^{2}$ JERRY R. MEYER, ${ }^{2}$ and EDWARD H. AIFER ${ }^{2}$ \\ 1.-U.S. Army Research Laboratory, RDRL-SEE-M, 2800 Powder Mill Road, Adelphi, MD 20783, \\ USA. 2.-U.S. Naval Research Laboratory, 4555 Overlook Ave. SW, Washington, DC 20375, USA. \\ 3.—e-mail: blair.connelly.ctr@mail.mil
}

\begin{abstract}
Time-resolved photoluminescence (TRPL) spectroscopy is used to study the minority-carrier lifetime in mid-wavelength infrared, $n$-type, InAs/Ga ${ }_{1-x} \mathrm{In}_{x} \mathrm{Sb}$ type II superlattices (T2SLs) and investigate the recombination mechanisms and trap states that currently limit their performance. Observation of multiple exponential decays in the intensity-dependent TRPL data indicates trap saturation due to the filling then emptying of trap states and different Shockley-Read-Hall (SRH) lifetimes for minority and majority carriers, with $\tau_{\text {maj }}\left(\tau_{n 0}\right) \gg \tau_{\min }\left(\tau_{p 0}\right)$. Simulation of the photoluminescence transient captures the qualitative behavior of the TRPL data as a function of temperature and excess carrier density. A trap state native to $\mathrm{Ga}_{1-x} \mathrm{In}_{x} \mathrm{Sb}$ is identified from the low-injection temperature-dependent TRPL data and found to be located below the intrinsic Fermi level of the superlattice, approximately $60 \pm 15 \mathrm{meV}$ above the valence-band maximum. Low-temperature TRPL data show a variation of the minority-carrier $\mathrm{SRH}$ lifetime, $\tau_{p 0}$, over a set of $\mathrm{InAs} / \mathrm{Ga}_{1-x} \mathrm{In}_{x} \mathrm{Sb} \mathrm{T} 2 \mathrm{SLs}$, where $\tau_{p 0}$ increases as $x$ is varied from 0.04 to 0.065 and the relative layer thickness of $\mathrm{Ga}_{1-x} \operatorname{In}_{x} \mathrm{Sb}$ is increased by $31 \%$.
\end{abstract}

Key words: Type II superlattice, minority-carrier lifetime, time-resolved photoluminescence, Shockley-Read-Hall, trap saturation, infrared

\section{INTRODUCTION}

Type II superlattice (T2SL) technology has the potential to surpass existing materials for use in mid-wavelength infrared (MWIR) and long-wavelength infrared (LWIR) photodetectors. ${ }^{1}$ The ability to engineer the bandgap promises low Auger recombination rates. ${ }^{2}$ In practice, however, InAs/ $\mathrm{Ga}_{1-x} \mathrm{In}_{x} \mathrm{Sb}$ T2SL material is limited by ShockleyRead-Hall (SRH) recombination, ${ }^{3-5}$ resulting in short minority-carrier lifetimes (tens of nanoseconds at $77 \mathrm{~K}$ ) that do not approach the theoretical limit determined by Auger recombination. A number of studies have been carried out to determine

(Received December 21, 2012; accepted August 23, 2013; published online September 25, 2013) the source of the SRH recombination center, including investigating the influences of varying the number of superlattice interfaces per unit length, ${ }^{6}$ surface recombination with varying absorber width, ${ }^{7}$ absorber doping level, ${ }^{8}$ and interface type. ${ }^{8}$ In each study, the varied superlattice parameter was found to have a negligible effect on the observed carrier lifetime. Therefore, it is postulated that a native defect in one of the superlattice constituents (InAs or $\mathrm{Ga}_{1-x} \operatorname{In}_{x} \mathrm{Sb}$ ) is the source of the dominant SRH recombination center. ${ }^{9}$ Recent measurements of "Ga-free" InAs/InAs ${ }_{1-x} \mathrm{Sb}_{x}$ T2SLs demonstrated an order-of-magnitude improvement in nonradiative carrier lifetime when Ga was eliminated. ${ }^{10,11}$ These results suggest that the SRH trap(s) limiting the carrier lifetime in InAs $/ \mathrm{Ga}_{1-x} \mathrm{In}_{x} \mathrm{Sb}$ T2SLs is native to the $\mathrm{Ga}_{1-x} \mathrm{In}_{x} \mathrm{Sb}$ layers. As the 
minority-carrier lifetime is a primary factor limiting the performance characteristics of T2SL detectors, it is important to investigate these recombination centers further to determine whether the lifetime can be improved.

In this work, trap saturation is observed in the transient photoluminescence (PL) signal from MWIR, $n$-type InAs $/ \mathrm{Ga}_{1-x} \operatorname{In}_{x} \mathrm{Sb}$ T2SLs, from which we conclude that the majority (electron) capture rate is significantly smaller than the minority (hole) capture rate. A trap state in the bottom half of the superlattice bandgap, approximately $60 \pm 15 \mathrm{meV}$ above the valence-band maximum, is identified. The low-temperature minority-carrier SRH lifetime is seen to vary dramatically across a set of InAs/ $\mathrm{Ga}_{1-x} \mathrm{In}_{x} \mathrm{Sb}$ T2SLs, where $\tau_{p 0}$ increases as $x$ is varied from 0.04 to 0.065 , and the fraction of the $\mathrm{Ga}_{1-x} \mathrm{In}_{x} \mathrm{Sb}$ layer thickness (relative to the superlattice period) is varied by $31 \%$.

\section{EXPERIMENTAL PROCEDURES}

Time-resolved photoluminescence (TRPL) was used to investigate a set of MWIR InAs/ $\mathrm{Ga}_{1-x} \operatorname{In}_{x} \mathrm{Sb}$ superlattice absorber samples. Details on the experimental approach can be found in Ref. 3. A 2- $\mu \mathrm{m}$ ultrafast laser pulse generates photoexcited carrier densities between $1 \times 10^{15} \mathrm{~cm}^{-3}$ and $1.2 \times 10^{17} \mathrm{~cm}^{-3}$ (pulse energies between $1 \mathrm{~nJ}$ and $120 \mathrm{~nJ}$ ). The initial excess carrier density was estimated using the measured beam diameter $(\sim 5 \mathrm{~mm})$ and pulse energy. An absorption coefficient of $2.3 \times 10^{4} \mathrm{~cm}^{-1}$ was assumed, ${ }^{12}$ which resulted in $\sim 90 \%$ absorption of the incident laser light by the superlattice absorber region. It should be noted that the low hole mobility observed in T2SLs will lead to nonuniform carrier densities in the vertical direction at low temperatures. ${ }^{13}$ This effect has been neglected here. PL from the sample was collected using reflective optics, and detected with a fast $\mathrm{HgCdTe}$ detector (3 ns temporal resolution). A closed-cycle helium cryostat was used to acquire intensity-dependent data at $11 \mathrm{~K}, 40 \mathrm{~K}, 77 \mathrm{~K}, 100 \mathrm{~K}, 125 \mathrm{~K}, 150 \mathrm{~K}$, $175 \mathrm{~K}, 200 \mathrm{~K}, 250 \mathrm{~K}$, and $300 \mathrm{~K}$. Low-injection data were acquired at $10-\mathrm{K}$ intervals between $140 \mathrm{~K}$ and $250 \mathrm{~K}$.

The T2SL absorber samples used in this study were grown using molecular-beam epitaxy on an $n$-type GaSb substrate, with a 0.5- $\mu$ m-thick buffer layer and a 100-A-thick InAs cap layer.
Unintentionally doped, 1- $\mu$ m-thick, InAs/ $\mathrm{Ga}_{1-x} \mathrm{In}_{x} \mathrm{Sb}$ T2SL absorber regions were sandwiched by two 100A-thick AlSb barriers to confine injected carriers to the absorber regions. The thicknesses and compositions of the various layers in the SLs are given in Table I. The InAs $/ \mathrm{Ga}_{1-x} \operatorname{In}_{x} \mathrm{Sb}$ layer thicknesses were varied between samples to target a $0.25 \mathrm{eV}$ bandgap, and PL measurements revealed bandgaps within $10 \mathrm{meV}$ of one another. The peak PL wavelengths were $4.93 \mu \mathrm{m}, 4.86 \mu \mathrm{m}$, and $5.05 \mu \mathrm{m}$ for SL1, SL2, and SL3, respectively. Hall measurements determined the absorber regions to be $n$-type $\left(\sim 10^{16} \mathrm{~cm}^{-3}\right)$.

\section{RESULTS AND DISCUSSION}

\section{Time-Resolved Photoluminescence Data}

Figure 1 plots the TRPL data taken for samples SL1 (a-d), SL2 (e-h), and SL3 (i-l) at $11 \mathrm{~K}$, $77 \mathrm{~K}, 150 \mathrm{~K}$, and $300 \mathrm{~K}$, respectively, for a series of optically generated excess carrier densities. At temperatures between $40 \mathrm{~K}$ and $175 \mathrm{~K}$, the PL signal for all three samples under high-injection conditions is seen to exhibit multiple exponential decay regions (see, for example, the PL decay for the highest excess carrier density in Fig. 1f). In the first few nanoseconds there is a very fast decay, followed by a slow decay for the next $50 \mathrm{~ns}$ to $100 \mathrm{~ns}$, then a faster decay followed by an even slower decay for times greater than $150 \mathrm{~ns}$. However, multiple exponential decay regions are not seen at the lowest injection levels. Instead, the TRPL signal demonstrates a substantially faster decay followed by the slower decay tail for times greater than $50 \mathrm{~ns}$.

Nonexponential behavior of recombination kinetics was first investigated theoretically by Blakemore,${ }^{14}$ who derived the effective steady-state SRH lifetime for an arbitrary injection level. Since modeling of the transient decay shows that the solution is nonexponential at high injection levels, it cannot be fit by a single lifetime and cannot be determined analytically. In the initial stages of decay, the time constants for excess electron and hole capture are determined by $\tau_{n 0}$ and $\tau_{p 0}$, respectively, and the dominating contribution is determined by the injection level. A deviation from the initial decay is observed at intermediate times, as the fraction of occupied traps shifts dramatically from the initial conditions. Eventually, the excess carrier density becomes sufficiently small that the SRH equation can be solved analytically. At these later times, the

Table I. Thicknesses and compositions for the three T2SL structures under study

\begin{tabular}{|c|c|c|c|c|}
\hline Structure & InAs Thickness (̊̊) & $\operatorname{Ga}_{1-x} \mathbf{I n}_{x}$ Sb Thickness (Å) & $x(\%)$ & $E_{\mathrm{g}}(\mathrm{meV})$ \\
\hline SL1 & 27.3 & 36.7 & 4 & 252 \\
\hline SL2 & 24.5 & 22.9 & 5 & 255 \\
\hline SL3 & 21.2 & 15.3 & 6.5 & 246 \\
\hline
\end{tabular}

The peak PL luminescence energy at $T=7 \mathrm{~K}$ is also given. 


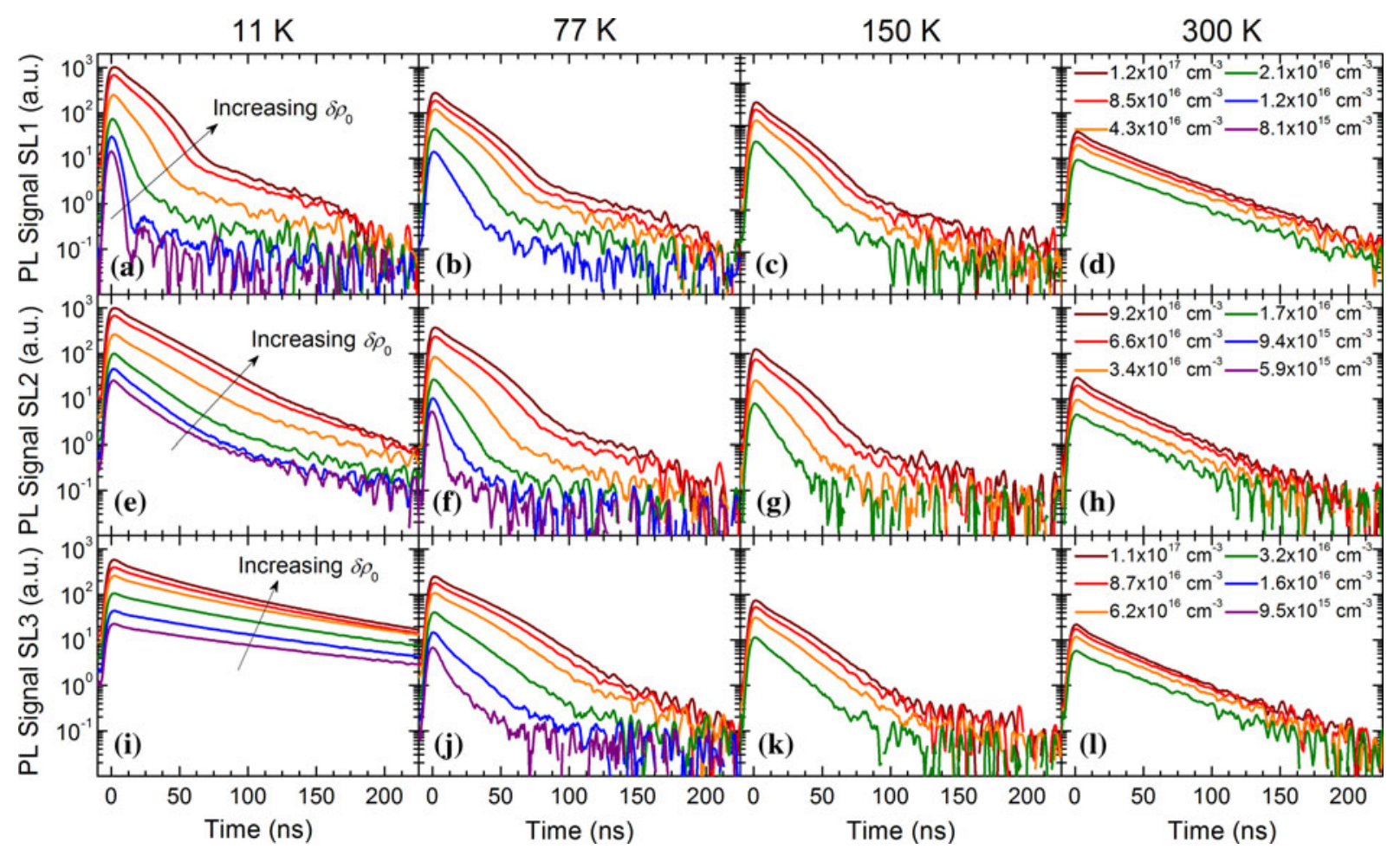

Fig. 1. Time-resolved photoluminescence data taken for samples (a-d) $S L 1\left(G_{0.96} \ln _{0.04} S b\right)$, (e-h) $S L 2$ (Gaa $\left.a_{0.95} I n_{0.05} S b\right)$, and (i-l) $S L 3$ $\left(\mathrm{Ga}_{0.935} \mathrm{In} \mathrm{n}_{0.065} \mathrm{Sb}\right)$ at $11 \mathrm{~K}, 77 \mathrm{~K}, 150 \mathrm{~K}$, and $300 \mathrm{~K}$, respectively, for a series of initial excess carrier densities, $\delta \rho_{0}$. In each plot, the peak PL signal at $t=0 \mathrm{~ns}$ increases with increasing $\delta \rho_{0}$. The curve with the largest peak PL signal corresponds to the largest initial excess carrier density in the legend(s) on the right.

photoluminescence decay approaches a quasi-steady-state decay and the recombination follows the longer of the two decay constants. The intensity dependence observed in GaAs and $\mathrm{Al}_{x} \mathrm{Ga}_{1-x} \mathrm{As}$ has been attributed to the saturation of trap states under high-injection conditions. ${ }^{15}$ Consequently, the decay deviates from the $\mathrm{SRH}$ recombination rate equation, which assumes steady-state capture of holes and electrons, and the resulting transient behavior varies with the initial excess carrier density. Under high injection, the carrier lifetime approaches the sum of the minority- and majoritycarrier lifetimes under both transient ${ }^{15}$ and steadystate conditions, ${ }^{16}$ whereas under low injection the traps do not saturate and recombination follows the minority-carrier lifetime. At intermediate injection levels, a transition between the two extremes is expected. For an $n$-type material, saturation of the recombination centers is expected to occur at injection levels where the ratio of initial excess carrier density to the background doping density exceeds the ratio of $\tau_{n 0}$ to $\tau_{p 0}{ }^{15}$ Further, the large variations we observe at $77 \mathrm{~K}$ and $150 \mathrm{~K}$ between the fast decay at low injection levels and the significantly longer decay at high injection suggests that the majority-carrier lifetime $\tau_{\text {maj }}\left(\tau_{n 0}\right)$ is significantly longer than the minority-carrier lifetime $\tau_{\min }\left(\tau_{p 0}\right)$. It is interesting to note that subtle evidence of trap saturation has been observed in a $p$-type, LWIR
(0.16 eV bandgap) InAs/GaSb T2SL for $T \leq 40 \mathrm{~K}^{3}$ In that case, the minority-carrier lifetime is the longer $\tau_{n 0}$.

\section{Low-Injection Lifetime}

The minority-carrier lifetime in sample SL3 was determined as a function of temperature, $T$, by fitting the low-injection TRPL data for excess carrier densities $<5 \times 10^{15} \mathrm{~cm}^{-3}$ with a single-exponential decay. Figure 2a plots the lifetime on a logarithmic scale as a function of $1000 / T$ for $T \geq 100 \mathrm{~K}$; Fig. $2 \mathrm{~b}$ plots the fit lifetimes as a function of temperature over a wider temperature range. The experimental lifetime decreases monotonically as the temperature increases from $11 \mathrm{~K}$ to $100 \mathrm{~K}$, which is consistent with the expected variation of SRH lifetime as $T^{-1 / 2}{ }^{17}$ In the low-temperature range, the observed lifetime is expected to follow this limiting value. ${ }^{16}$ As the temperature increases from $100 \mathrm{~K}$ to $250 \mathrm{~K}$, however, the increasing population of thermally generated minority carriers causes the lifetime to increase for as long as the sample remains in the extrinsic range. ${ }^{16}$ Above $250 \mathrm{~K}$, the lifetime again decreases as the sample becomes intrinsic.

Hall observed that the trap energy, $E_{\mathrm{t}}$, can be determined from the slope of $\ln (\tau)$ as a function of $1 / T$ in the high-temperature extrinsic range. ${ }^{16} \mathrm{~A}$ linear fit to $\ln (\tau)=\Delta E / k T+C$ provides the relative 


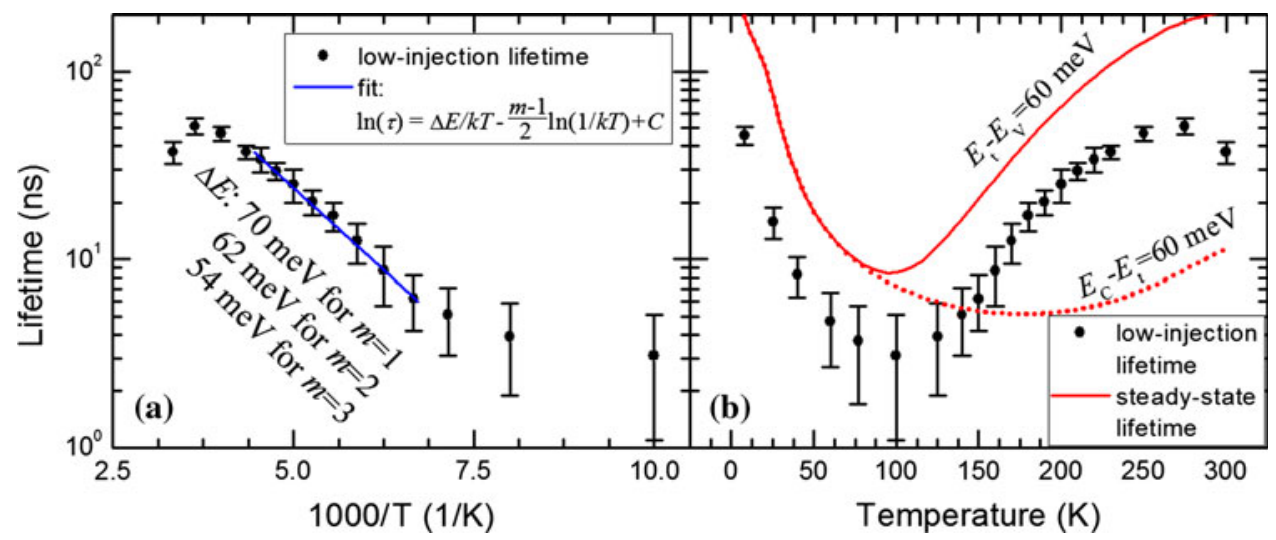

Fig. 2. Fits using a single-exponential decay for the carrier lifetime at low injection [points in (a) and (b)] for sample SL3. (a) Lifetime data as a function of $1000 / T$ fit with the function $\ln (\tau)=\Delta E / k T+C$ (blue line). The $\Delta E=60 \pm 15 \mathrm{meV}$ slope gives the position of the trap state above the valence band (or below the conduction band). (b) Modeled steady-state Shockley-Read-Hall lifetime (red line) as a function of temperature using a trap energy of $60 \mathrm{meV}$ above the valence band (Color figure online).

position of the trap state, $\Delta E$, either above the valence-band maximum, $E_{\mathrm{v}}$, or below the conduction-band minimum, $E_{\mathrm{C}}$. Here, $k$ is the Boltzmann constant and $C$ is a constant. Since the samples studied here possess a narrow bandgap, the temperature dependence of the terms comprising $C$ can be important. If the excess carrier density is neglected for low injection levels, the SRH lifetime ${ }^{18}$ can be reduced to

$$
\ln (\tau)=\frac{\Delta E}{k T}-\frac{m-1}{2} \ln \left(\frac{1}{k T}\right)+C
$$

where the $(m / 2) \ln (1 / k T)$ contribution is derived from the temperature dependence of the density of states of the valence (or conduction) band, with $m$ representing the dimensionality of the density of states. Here, $2 \leq m \leq 3$ with $m$ approaching 3 at high temperatures. The $(-1 / 2) \ln (1 / k T)$ contribution is derived from the $T^{-1 / 2}$ dependence of $\tau_{n 0}$ (or $\left.\tau_{p 0}\right)$ due to the temperature dependence on thermal velocity. Neglecting the $\ln (1 / k T)$ term will lead to an error of $k T$ in the determination of $\Delta E$, which is approximately $16 \mathrm{meV}$ here. Therefore, when $\Delta E$ is large compared with $k T$, that term can be neglected. However, for narrow-bandgap materials (or shallow traps), the $\ln (1 / k T)$ term is important. Figure $2 \mathrm{a}$ plots the fit to the low-injection lifetime using Eq. 1 (solid blue line). Neglecting the $\ln (1 / k T)$ term gives the result $\Delta E=70 \mathrm{meV}$; including the $\ln (1 / k T)$ term and assuming $m=2$ (3) gives the result $\Delta E=62 \mathrm{meV}$ (54 meV). The fast decay for temperatures between $60 \mathrm{~K}$ and $150 \mathrm{~K}$ is the result of the convolution of a fast time constant with the $\sim 3 \mathrm{~ns}$ system response time, resulting in fit lifetimes that are likely overpredicting the carrier lifetime. Accounting for the system response would increase the estimate for $\Delta E$, and since the dimensionality changes with temperature, the average trap energy of $\Delta E=60 \pm 15 \mathrm{meV}$, either above $E_{\mathrm{v}}$ or below $E_{\mathrm{C}}$, is utilized here.
The modeled temperature-dependent steady-state SRH lifetime for a trap position $60 \mathrm{meV}$ above the valence band is plotted in Fig. $2 \mathrm{~b}$ (red line). The qualitative agreement with the experimental lifetime is seen to be quite good in all three of the temperature regions discussed above, i.e., $\leq 100 \mathrm{~K}$ where the lifetime decreases, $100 \mathrm{~K}$ to $250 \mathrm{~K}$ where it increases, and $\geq 250 \mathrm{~K}$ where it again decreases. Conversely, assuming a trap position $60 \mathrm{meV}$ below the conduction band ( $190 \mathrm{meV}$ above the valence band for a $250 \mathrm{meV}$ bandgap) leads to poor agreement with the experimental temperature dependence, since the minimum from the model then occurs at $\sim 180 \mathrm{~K}$, with a slower increase in lifetime as $T$ is increased than what is observed experimentally. The offset between the experimental and modeled lifetimes in Fig. $2 \mathrm{~b}$ can be explained by the different time scales that dominate each quantity. The low-injection TRPL lifetime is dominated by the minority-carrier SRH lifetime, $\tau_{p 0}$. For a trap position different from the intrinsic Fermi level, however, the steady-state SRH lifetime depends on both the majority- and minority-carrier SRH lifetimes. The decrease in lifetime with increasing temperature at low temperatures is seen to vary much faster than the theoretically predicted $T^{-1 / 2}$ dependence. This is likely due to the freeze-out of electrons at low temperatures.

The estimation of $E_{\mathrm{t}}=60 \pm 15 \mathrm{meV}$ is reinforced by observing the dependence of the carrier lifetime on excess carrier density. For $T \leq 180 \mathrm{~K}$, the lifetime is seen to increase with increasing carrier density; for $T \geq 190 \mathrm{~K}$, the lifetime is seen to decrease with increasing carrier density. This observation requires ${ }^{14}$

$$
\frac{1}{n_{0}+p_{0}}=\frac{\tau_{p 0}+\tau_{n 0}}{\tau_{p 0}\left(n_{0}+n_{1}\right)+\tau_{n 0}\left(p_{0}+p_{1}\right)}
$$

at $\sim 185 \mathrm{~K}$, where $n_{0}$ and $p_{0}$ are the background electron and hole density, respectively, and $n_{1}$ is the 
number of electrons in the conduction band and $p_{1}$ is the number of holes in the valence band when the Fermi level coincides with $E_{\mathrm{t}}$. Using the approximations $p_{0}=0$ and $\tau_{n 0} \gg \tau_{p 0}$ results in a trap energy of $\sim 60 \mathrm{meV}$ for an effective hole mass of $\sim 0.1 m_{0}$, where $m_{0}$ is the electron rest mass. The effective hole mass will influence the density of states of the valence band, and therefore affect the thermal population of the trap state as temperature is increased. Fitting Eq. 1 assumes that the electron and hole capture cross-sections do not exhibit any activation behavior besides the $T^{-1 / 2}$ dependence on the thermal velocity accounted for by the temperature dependence of $\tau_{p 0}$.

Equation 2 is expected to be valid when the Fermi level coincides with the trap position, providing another good verification of the trap energy. Calculations of the superlattice band structure provide an approximate Fermi energy of $80 \pm 3 \mathrm{meV}$ above the valence-band maximum at $185 \mathrm{~K}$. It can therefore be expected that Eq. 2 is valid for a trap energy of $80 \mathrm{meV}$, providing reasonable agreement with the experimentally obtained trap energy of $\Delta E=$ $60 \pm 15 \mathrm{meV}$. Note that a trap energy of $80 \mathrm{meV}$ using Eq. 2 corresponds to an effective hole mass of $0.25 m_{0}$.

\section{Transient Photoluminescence Simulations}

The transient decay of the PL signal from a shortpulse excitation can vary significantly from the behavior predicted by the steady-state SRH equation. To understand this behavior better, we modeled the dynamics of filling and emptying of a SRH trap state under optical excitation by a subpicosecond pulse. Since this analysis considers an $n$-type sample, the majority/minority carriers will be referred to as electrons/holes, though an analogous treatment may be deduced for the case of a $p$-type sample. The simulated PL transient for a series of initial excess carrier densities is plotted in Fig. 3 at (a) $11 \mathrm{~K}$, (b) $77 \mathrm{~K}$, (c) $150 \mathrm{~K}$, and (d) $300 \mathrm{~K}$. Also plotted are other relevant parameters (excess electrons, excess holes, and occupied traps), along with the simulated PL transient, for four conditions: a case where trap saturation occurs at $77 \mathrm{~K}$ (Fig. 4a, b) and a case where trap saturation does not occur at $300 \mathrm{~K}$ (Fig. 4c, d), for both high (a, c) and low $(b, d)$ injection.

Above $200 \mathrm{~K}$, trap saturation does not occur and a simple exponential decay is observed (Fig. 3d), because the trap state is always filled with electrons and the filling and emptying of the trap state is unimportant. The simulations at $300 \mathrm{~K}$ (Fig. 4c, d) demonstrate that the density of occupied traps does not deviate significantly from initial conditions, the excess hole and electron densities are nearly equal, and the transient decay directly follows the decay of the excess carriers.

Below $200 \mathrm{~K}$, the filling and emptying of the trap state becomes important. Under low injection, where the excess carrier density is significantly smaller than the $\sim 10^{16} \mathrm{~cm}^{-3}$ background doping density, a faster decay is observed, which is determined by the hole capture rate. This behavior is explained by the feature in Fig. $4 \mathrm{~b}$ that the occupied trap density does not change significantly, hence the simulated PL transient for $t<50 \mathrm{~ns}$ directly follows the decay of the minority excess holes. For $t>50 \mathrm{~ns}$, the simulated PL transient follows the decay of the majority excess electrons and, as a consequence, the capture of excess holes follows this same rate. When the excess carrier density is comparable to the background doping density, trap saturation is observed in the multiple decay regions of the simulated PL signal, which can be seen for injection levels above $10^{16} \mathrm{~cm}^{-3}$ at $77 \mathrm{~K}$ and $150 \mathrm{~K}$ (Fig. 3b, c) and subtly at $11 \mathrm{~K}$ (Fig. 3a). The highinjection case is illustrated in Fig. $4 \mathrm{a}$ at $77 \mathrm{~K}$. The fast initial decay, in the first few nanoseconds, corresponds to emptying of the trap state by electrons at the hole capture rate. Once the traps are empty, the recombination rate is determined by the capture rate of excess majority electrons. Here we assume that the electron capture cross-section is smaller than that of the holes (with a ratio of $\sim 0.05$ ), resulting in a slower decay since the carrier recombination rate is limited by electron capture. Note here that, for $t<50 \mathrm{~ns}$, the decay rate for excess holes and the simulated PL transient follow the decay of excess electrons, and the trap occupation increases at a comparable rate. As the traps begin to fill with electrons, a faster decay is observed, being mainly determined by the hole capture rate. Finally, the decay approaches the rate determined by the SRH equation at sufficiently low excess carrier density where the occupation of trap states reaches its quasi-steady-state condition, which is dominated by the slower capture of excess electrons.

\section{Variation with Superlattice Composition}

Comparison of the TRPL data for samples SL1, SL2, and SL3 at $11 \mathrm{~K}$ in Fig. 1 reveals drastically different behavior. SL1 (Fig. 1a) exhibits strong trap saturation behavior at $11 \mathrm{~K}$, where a very fast decay at the lowest injection level becomes a longer decay with the characteristic bowing behavior at the highest injection; SL2 (Fig. 1e) shows only subtle bowing for the three highest injection levels plotted; SL3 (Fig. 1i) does not demonstrate any bowing of the PL signal. SL3 is observed to exhibit bowing in the TRPL decay at high injections at $40 \mathrm{~K}$. To make a direct comparison between the three samples at $11 \mathrm{~K}$, the high-injection data were normalized with respect to each other and are plotted in Fig. 5a. The overall decay times vary significantly between the three samples, with SL3 having the longest, SL2 the middle, and SL1 the most rapid decay. This trend correlates with both changes in layer thicknesses and the alloy composition of the $\mathrm{Ga}_{1-x} \mathrm{In}_{x} \mathrm{Sb}$ 


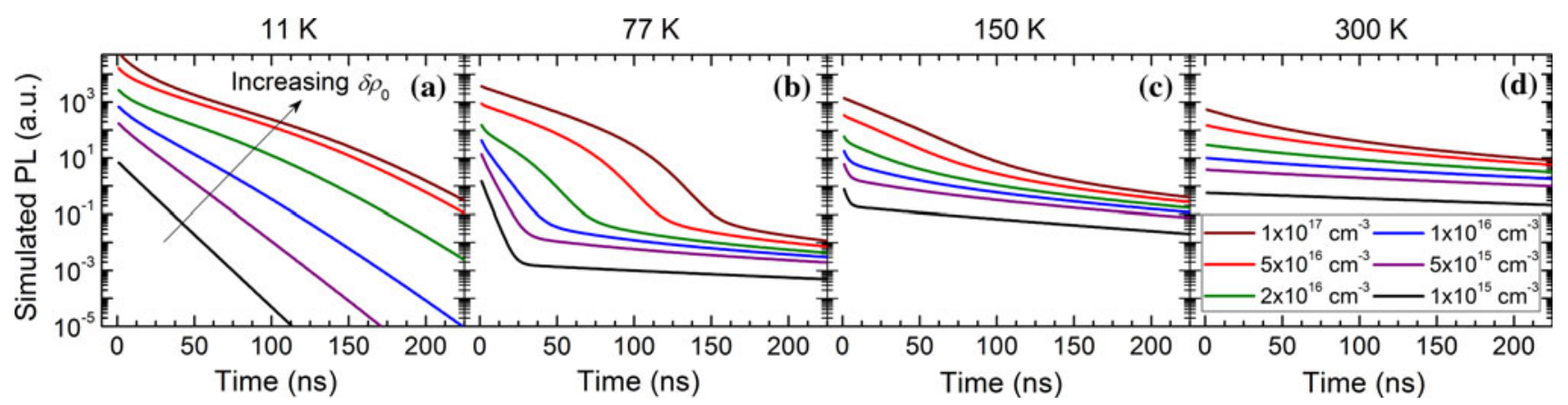

Fig. 3. Simulated transient photoluminescence at (a) $11 \mathrm{~K}$, (b) $77 \mathrm{~K}$, (c) $150 \mathrm{~K}$, and (d) $300 \mathrm{~K}$ for a series of initial excess carrier densities.

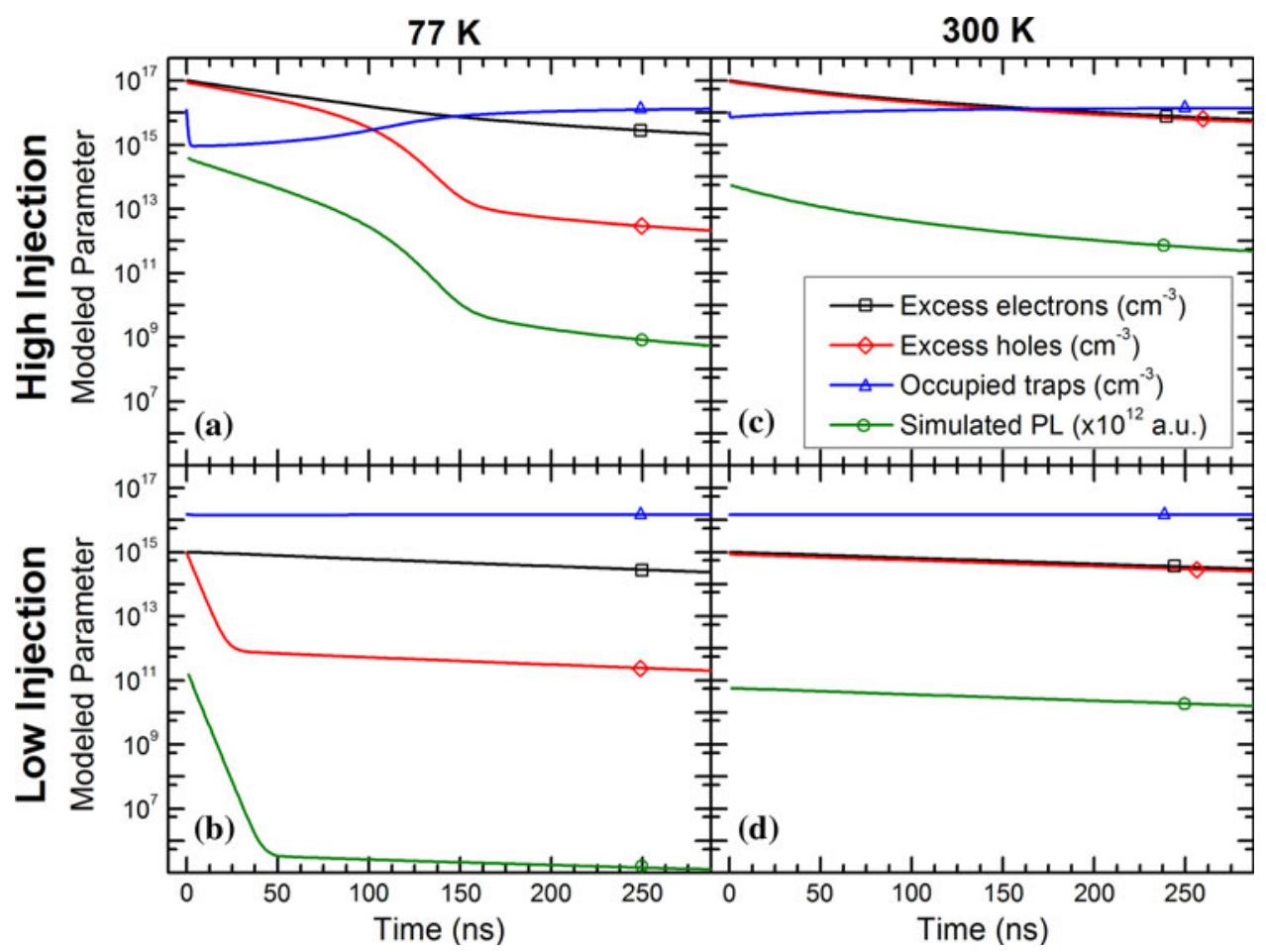

Fig. 4. Simulated transient of excess electrons (black line, square), excess holes (red line, diamond), occupied traps (blue line, triangle), and simulated photoluminescence (green line, circle) for cases: (a) at $77 \mathrm{~K}$ under high injection $\left(10^{17} \mathrm{~cm}^{-3}\right)$, (b) at $77 \mathrm{~K}$ under low injection $\left(10^{15} \mathrm{~cm}^{-3}\right)$, (c) at $300 \mathrm{~K}$ under high injection $\left(10^{17} \mathrm{~cm}^{-3}\right)$, and (d) at $300 \mathrm{~K}$ under low injection $\left(10^{15} \mathrm{~cm}^{-3}\right)$ (Color figure online).

layer of the superlattice (Table I), where the decay time is seen to increase with both increasing hole well thickness and with increasing $x$. The presence of strong bowing behavior is most closely related to a short $\tau_{p 0}$ relative to $\tau_{n 0}$; i.e., as $\tau_{p 0}$ decreases, the prominence of bowing in the TRPL decay is expected to increase. As an illustration, the simulated PL transient is plotted in Fig. 5b for a series of trap densities, $N_{\mathrm{t}}$, between $5 \times 10^{15} \mathrm{~cm}^{-3}$ and $1 \times 10^{17} \mathrm{~cm}^{-3}$ and the simulated excess electron density (solid lines) and excess hole density (dotted lines) is plotted in Fig. 5c for trap densities of $1 \times 10^{16} \mathrm{~cm}^{-3}$, $2 \times 10^{16} \mathrm{~cm}^{-3}$, and $4 \times 10^{16} \mathrm{~cm}^{-3}$. Clearly, a higher trap density results in a shorter decay time due to a shorter $\tau_{p 0}$, as well as a more prominent contribution of trap saturation to the transient sig- nal. For trap densities $\left(N_{\mathrm{t}}\right)$ below $1 \times 10^{16} \mathrm{~cm}^{-3}$, trap saturation has a negligible effect on the decay and bowing is not observed, because $\tau_{p 0}$ is longer. Such behavior is consistent with the decay observed for SL3. As $N_{\mathrm{t}}$ is increased, $\tau_{p 0}$ decreases and the bowing behavior associated with trap saturation begins to appear, which corresponds to the behavior observed for SL2. As $N_{\mathrm{t}}$ becomes large $\left(N_{\mathrm{t}}>3 \times 10^{16} \mathrm{~cm}^{-3}\right)$, the behavior observed in SL1 appears and the decay is dominated by trap saturation.

Increases in the trap density due to changes in superlattice composition are not solely responsible for the observed changes in TRPL decay at $11 \mathrm{~K}$. In order to capture the trend observed between SL1 and SL3, $N_{\mathrm{t}}$ is varied by a factor of 4 in Fig. 5b, while $x$ only varies between $4 \%$ and $6.5 \%$ and the 


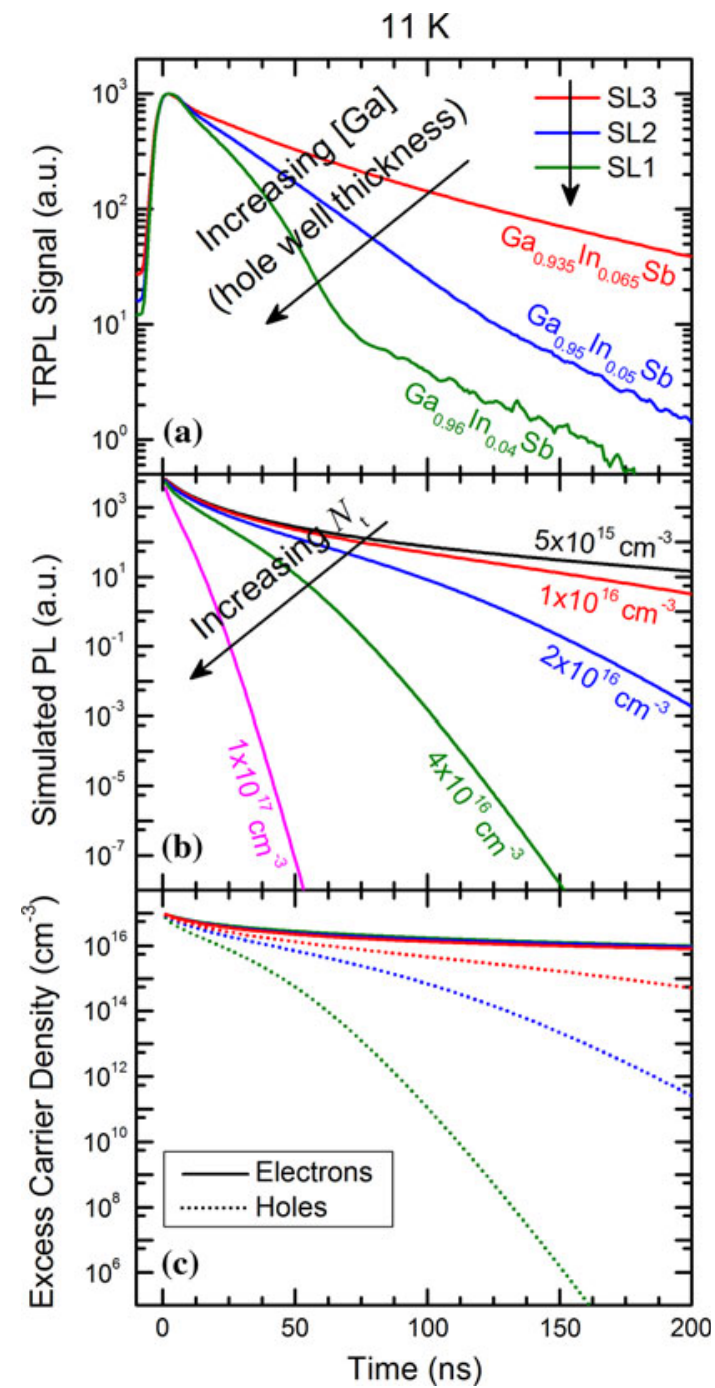

Fig. 5. (a) Normalized time-resolved photoluminescence signal in a set of $\mathrm{Ga}_{1-x} \mathrm{In}_{x} \mathrm{Sb}$ superlattices at $11 \mathrm{~K}$ under high injection $\left(\sim 10^{17} \mathrm{~cm}^{-3}\right)$. (b) Simulated transient photoluminescence under high injection $\left(10^{17} \mathrm{~cm}^{-3}\right)$ for a series of trap densities, $N_{\mathrm{t}}$, between $5 \times 10^{15} \mathrm{~cm}^{-3}$ and $1 \times 10^{17} \mathrm{~cm}^{-3}$. (c) Simulated excess electron (solid lines) and excess hole (dotted lines) density for $N_{t}$ of $1 \times 10^{16} \mathrm{~cm}^{-3}, 2 \times 10^{16} \mathrm{~cm}^{-3}$, and $4 \times 10^{16} \mathrm{~cm}^{-3}$.

thickness of the $\mathrm{Ga}_{1-x} \mathrm{In}_{x} \mathrm{Sb}$ layer (hole well) relative to the superlattice period varies by $31 \%$. Calculations show that the valence-band offset also changes as the superlattice composition is changed, with the position of the valence band of SL3 being $>100 \mathrm{meV}$ lower than the position of the valence band of SL1 (relative to the $\mathrm{Ga}_{1-x} \mathrm{In}_{x} \mathrm{Sb}$ valence band). If we assume that the trap level is relatively insensitive to quantum confinement, the trap position observed in SL3 could then correspond to a trap below the valence-band edge in SL1, suggesting that the observed lifetimes could be controlled by three different traps in the three superlattices studied. Further, it is possible that the effect of changing the valence-band offset can also affect $\tau_{p 0}$, and when combined with an increasing trap density with an increase of the fractional $\mathrm{Ga}_{1-x} \mathrm{In}_{x} \mathrm{Sb}$ layer thickness per superlattice period (along with other effects), $\tau_{p 0}$ can be varied by the factor of 4 observed experimentally.

\section{CONCLUSIONS}

Time-resolved photoluminescence was used to identify the approximate energy of a trap state in a MWIR T2SL absorber sample. The multiple exponential decay regions observed in the TRPL data are attributed to the filling and then emptying of trap states, combined with different $\mathrm{SRH}$ lifetimes for minority and majority carriers: $\tau_{\text {maj }}\left(\tau_{n 0}\right) \gg \tau_{\min }\left(\tau_{p 0}\right)$. The trap state is found to be located below the Fermi level for the $n$-type samples, and is expected to be a native defect occurring in the $\mathrm{Ga}_{1-x} \mathrm{In}_{x} \mathrm{Sb}$ layer of the superlattice. A relatively shallow trap position of $60 \pm 15 \mathrm{meV}$ above the valence band is identified as the dominant SRH recombination center for a MWIR T2SL. Low-temperature TRPL data show a variation of the minority-carrier $\mathrm{SRH}$ lifetime, $\tau_{p 0}$, over a set of InAs $/ \mathrm{Ga}_{1-x} \mathrm{In}_{x} \mathrm{Sb}$ T2SLs, where $\tau_{p 0}$ increases as $x$ is varied from 0.04 to 0.065 and the relative layer thickness of $\mathrm{Ga}_{1-x} \mathrm{In}_{x} \mathrm{Sb}$ is increased by $31 \%$. For T2SL detector applications, $p$-type superlattices are more commonly used, and their performance is dependent on the minority-carrier lifetime, $\tau_{n 0}$. Therefore, the longer time constant associated with $\tau_{n 0}$ is expected to be important for T2SL detectors.

\section{ACKNOWLEDGEMENTS}

B.C.C. would like to acknowledge research supported in part by an appointment to the U.S. Army Research Laboratory Postdoctoral Fellowship Program administered by the Oak Ridge Associated Universities through a cooperative agreement.

\section{REFERENCES}

1. M.A. Kinch and A. Yariv, Appl. Phys. Lett. 55, 2093 (1989).

2. C.H. Grein, H. Cruz, M.E. Flatte, and H. Ehrenreich, Appl. Phys. Lett. 65, 2532 (1994).

3. B.C. Connelly, G.D. Metcalfe, H. Shen, and M. Wraback, Appl. Phys. Lett. 97, 251117 (2010).

4. D. Donetsky, S.P. Svensson, L.E. Vorobjev, and G. Belenky, Appl. Phys. Lett. 95, 212104 (2009).

5. J. Pellegrino and R. DeWames, Proc. SPIE 7982, 72981U (2009).

6. S.P. Svensson, D. Donetsky, D. Wang, P. Maloney, and G. Belenky, Proc. SPIE 7660, 76601V (2010).

7. B.C. Connelly, G.D. Metcalfe, H. Shen, and M. Wraback, IJHSES 20, 541 (2011).

8. B.C. Connelly, G.D. Metcalfe, H. Shen, and M. Wraback, Proc. SPIE 8155, 81550L (2011).

9. S.P. Svensson, D. Donetsky, D. Wang, H. Hier, F.J. Crowne, and G. Belenky, J. Cryst. Growth 334, 103 (2011).

10. E.H. Steenbergen, B.C. Connelly, G.D. Metcalfe, H. Shen, M. Wraback, D. Lubyshev, Y. Qiu, J.M. Fastenau, A.W.K. Liu, S. Elhamri, O.O. Cellek, and Y.H. Zhang, Appl. Phys. Lett. 99, 251110 (2011).

11. B.V. Olson, E.A. Shaner, J.K. Kim, J.F. Klem, S.D. Hawkins, L.M. Murray, J.P. Prineas, M.E. Flatté, and T.F. Boggess, Appl. Phys. Lett. 101, 092109 (2012). 
12. J.W. Little, S.P. Svensson, W.A. Beck, A.C. Goldberg, S.W. Kennerly, T. Hongsmatip, M. Winn, and P. Uppal, J. Appl. Phys. 101, 044514 (2007).

13. B.V. Olson, L.M. Murray, J.P. Prineas, M.E. Flatté, J.T. Olesberg, and T.F. Boggess, Appl. Phys. Lett. 102, 202101 (2013).

14. J.S. Blakemore, Semiconductor Statistics, 1st ed. (New York: Pergamon, 1962), pp. 250-304.
15. R.K. Ahrenkiel, B.M. Keyes, and D.J. Dunlavy, J. Appl. Phys. 70, 225 (1991).

16. R.N. Hall, Phys. Rev. 87, 387 (1952).

17. R.K. Ahrenkiel, Minority Carriers in III-V Semiconductors: Physics and Applications, Vol. 39, ed. R.K. Ahrenkiel and M.S. Lundstrom (New York: Academic Press, Inc., 1993), pp. 39-150.

18. W. Shockley and W.T. Read, Phys. Rev. 87, 835 (1952). 